\title{
Modelagem comparativa da cinética da fase sólida do capim-elefante picado, suplementado ou não com concentrados, e fornecido a vacas Holandês x Zebu em lactação
}

\author{
[Comparative modeling of chopped elephantgrass particulate kinectics in crossbred lactating \\ cows receiving or not concentrate supplement] \\ F.C.F. Lopes ${ }^{1}$, J.S. Oliveira ${ }^{1}$, D.S.C. Paciullo ${ }^{1}$, L.J.M. Aroeira ${ }^{1}$, F. Deresz ${ }^{1}$, M.J.F. Morenz ${ }^{2}$, \\ A.C.A. Duque , A.C.W. Elyas ${ }^{3}$ \\ ${ }^{1}$ Embrapa Gado de Leite \\ Rua Eugênio do Nascimento, 610 \\ 36038-330 - Juiz de Fora, MG \\ ${ }^{2}$ Instituto de Zootecnia - UFRRJ - Seropédica, RJ \\ ${ }^{3}$ Aluno de graduação - CES - Juiz de Fora, MG \\ ${ }^{4}$ Aluno de pós-graduação - UFLA - Lavras, MG
}

\begin{abstract}
RESUMO
Foram comparadas as estimativas da cinética da fase sólida do capim-elefante (Pennisetum purpureum, Schum.) picado, mordentado com cromo $(\mathrm{Cr})$, obtidas do ajuste de dois modelos não-lineares (bicompartimental tempo-independente e multicompartimental tempo-dependente) a resultados de excreção fecal de vacas Holandês x Zebu em lactação. Utilizaram-se dados de dois experimentos realizados em anos diferentes com capim-elefante cortado aos 60 e 45 dias, e suplementado, $3,3 \mathrm{~kg} / \mathrm{vaca} / \mathrm{dia}$, base matéria natural, ou não com concentrados. Foram utilizadas quatro e três vacas, respectivamente, no primeiro e no segundo ano de experimentação, havendo sempre duas fases de coleta de dados, necessárias para permitir que cada vaca pudesse ser avaliada em cada tratamento. As taxas de passagem ruminal estimadas variaram de 3,0 a $3,2 \% / \mathrm{h}$ no modelo bicompartimental tempo-independente, e de 2,6 a $3,0 \% / \mathrm{h}$ no modelo multicompartimental tempo-dependente, enquanto as respectivas taxas de passagem pós-ruminal variaram de 4,9 a 7,4\%/h e de 7,4 a $10,9 \% / \mathrm{h}$. Os tempos médios de retenção do capim-elefante no trato gastrintestinal das vacas variaram de 66,0 a $76,2 \mathrm{~h}$ no modelo bicompartimental tempo-independente, e de 48,1 a 57,8h no modelo multicompartimental tempo-dependente. O processo de dependência de tempo imposto pelo modelo multicompartimental permitiu melhor ajuste aos dados de excreção fecal do $\mathrm{Cr}$, em relação ao modelo bicompartimental.
\end{abstract}

Palavras-chave: bovino, indicador, mordente, Pennisetum purpureum, taxa de passagem

\begin{abstract}
Particulate kinetics estimates of chopped elephantgrass (Pennisetum purpureum, Schum.) mordanted with Chromium (Cr) were obtained and compared by the adjustment of two nonlinear models - ageindependent double-compartmental model and age-dependent multicompartmental model - to a Cr faecal excretion dates of lactating crossbred Holstein $x$ Zebu cows. Results from two trials carried out in different years with elephantgrass cut at 60 and 45 days of growth and supplemented, $3,3 \mathrm{~kg} / \mathrm{cow} /$ day, wet basis, or not with concentrates were used. Four cows in the first and three cows in the second year were used and in each year there were two phases of collection of data, necessary to allow that each cow could be evaluated in each treatment. The ruminal particulate passage rates of chopped elephantgrass ranged from 3.0 to $3.2 \% / \mathrm{h}$ in age-independent double-compartmental model, and from 2.6 to $3.0 \% / \mathrm{h}$ in agedependent-multicompartmental model, while that the respective post-ruminal passage rates ranged from
\end{abstract}

Recebido em 24 de agosto de 2006

Aceito em 4 de abril de 2008

E-mail: fernando@cnpgl.embrapa.br 
4.9 to $7.4 \% / h$ and from 7.4 to $10.9 \% / h$. The total mean retention time of the elephantgrass in the gastrointestinal tract ranged from 66.0 to $76.2 \mathrm{~h}$ in age-independent double-compartmental model, and from 48.1 to $57.8 \mathrm{~h}$ in age-dependent-multicompartmental model. The implicit assumption of agedependence for the multicompartimental model allowed better fit to the data of faecal excretion $\mathrm{Cr}$ than that one of the double-compartmental model.

Keywords: Cattle, chromium, fractional outflow rate, marker, mordant, Pennisetum purpureum

\section{INTRODUÇÃO}

A taxa de passagem no rúmen é uma variável que afeta a utilização de nutrientes, haja vista modular o tempo disponível para os processos de digestão e absorção (Ehle, 1984), sendo, por isso, importante em modelos matemáticos de formulação de dietas, avaliação e predição de desempenho de rebanhos (Nutrient..., 2001; Fox et al., 2004). Ademais, por estar diretamente relacionada ao efeito de enchimento físico do rúmen, influencia o consumo voluntário de alimentos e, por conseguinte, a produtividade animal (Van Soest, 1994).

Existem diversos procedimentos metodológicos para obtenção de estimativas dessa variável (Lascano e Quiroz, 1990). Em detrimento às excessivamente laboriosas metodologias in vivo, técnicas indiretas, com o uso de indicadores nãoabsorvíveis, ingeridos ou diretamente infundidos no rúmen em dose única, têm sido mais adotadas nas estimativas dos parâmetros de dinâmica da passagem das partículas no trato gastrintestinal (TGI) dos ruminantes (Offer e Dixon, 2000).

Dentre vários indicadores utilizados nas estimativas de taxa de passagem da fase sólida (Van Soest, 1994; Offer e Dixon, 2000; Lopes, 2002), foi demonstrado que as fibras complexadas com cromo $(\mathrm{Cr})$, denominadas de cromo-mordentes, foram eficientes na representação do fluxo de partículas no TGI de novilhos (Lira et al., 2006).

Conforme revisão de literatura apresentada por Lopes (2002), vários são os modelos não-lineares para ajuste das curvas da concentração fecal do indicador, em função do tempo transcorrido desde sua administração, visando à determinação dos parâmetros da dinâmica da passagem das partículas no TGI.

Para condições tropicais, o modelo de Dhanoa et al. (1985) apresentou melhor capacidade de ajuste aos dados de excreção fecal de indicadores com bovinos mantidos sob estabulação (Oliveira et al., 1999) ou em condição de pastejo (Lira et al., 2006), quando comparado ao modelo de Grovum e Williams (1973). Para vacas em lactação manejadas em pastagem de capimelefante, não foi observada superioridade de um modelo em relação ao outro no ajuste aos dados de excreção fecal de cromo (Lopes et al., 2003).

O objetivo deste trabalho foi comparar dois modelos não-lineares no ajuste de curvas de excreção fecal do cromo de vacas Holandês x Zebu em lactação que receberam capim-elefante picado, suplementado ou não com concentrados.

\section{MATERIAL E MÉTODOS}

Foram utilizados dados de excreção fecal de cromo de dois experimentos realizados no Campo Experimental de Coronel Pacheco, de propriedade da Embrapa Gado de Leite. Os experimentos foram realizados em anos diferentes, utilizando capim-elefante com idades de corte de 60 (primeiro ano) ou 45 dias (segundo ano), fornecido picado a vacas Holandês x Zebu em lactação, canuladas no rúmen. Foram utilizadas quatro e três vacas, respectivamente, no primeiro e no segundo ano de experimentação. Em cada ano de avaliação, houve duas fases de coleta de dados, necessárias para permitir que cada vaca pudesse ser avaliada em cada tratamento (Lopes et al., 1997). Foi observado período de adaptação às dietas experimentais nunca inferior a 15 dias.

As vacas, em média, com $470 \mathrm{~kg}$ de peso corpóreo e produção de 4 a $8 \mathrm{~kg} /$ dia de leite permaneceram confinadas em baias individuais, onde receberam, em duas refeições diárias, as dietas experimentais: 1) capim-elefante como alimento exclusivo, e 2) capim-elefante suplementado com 3,3kg/vaca $/$ dia de concentrado (base matéria natural), formulado à base de $52 \%$ de milho triturado e $48 \%$ de farelo de algodão, fornecido em duas porções diárias, em cocho separado. O concentrado utilizado para 
suplementação do capim-elefante apresentou, em média, $84 \%$ de matéria seca (MS), 23,8\% de proteína bruta $(\mathrm{PB})$ e um valor estimado de $75 \%$ de nutrientes digestíveis totais. A composição química do capim-elefante picado, cortado com 60 (primeiro ano) e 45 dias de idade (segundo ano) apresentou, em média, respectivamente, 18,7 e $17,2 \%$ de MS; 10,1 e $10,6 \%$ de PB; 72,0 e $69,9 \%$ de fibra em detergente neutro (FDN); e 41,2 e $42,7 \%$ de fibra em detergente ácido (FDA).

Em cada fase experimental de cada ano, foram coletadas amostras de capim-elefante picado, que foram submetidas à extração à quente com detergente neutro comercial, da fração FDN que, posteriormente, foi complexada com dicromato de sódio $\left(\mathrm{Na}_{2} \mathrm{Cr}_{2} \mathrm{O}_{7} .2 \mathrm{H}_{2} \mathrm{O}\right)$, conforme procedimentos relatados por Úden et al. (1982). Em cada fase experimental, foram administrados, via rúmen das vacas, $100 \mathrm{~g}$ (primeiro ano) e $90 \mathrm{~g}$ (segundo ano) de FDN de capim-elefante mordentada com cromo.

No primeiro ano, foram realizadas coletas de fezes às $6,10,14,18,22,26,30,33,36,39,42$, $45,48,52,56,60,64,68,72,78,84,90,96,102$, 108 e 120h após a administração do indicador e, no segundo ano, às $6,10,14,18,22,25,28,31$, $34,37,40,44,48,52,56,60,64,68,72,78,84$, $90,96,102,108,114$ e $120 \mathrm{~h}$.

As amostras individuais de fezes foram pré-secas por $72 \mathrm{~h}$ em estufa de ventilação forçada, regulada para $65^{\circ} \mathrm{C}$, sendo, posteriormente, moídas em moinho de facas dotado de peneira com abertura de malhas de $1 \mathrm{~mm}$. A seguir, foram analisadas para teor de $\mathrm{Cr}$ por espectrofotometria de absorção atômica, após digestão nitroperclórica, conforme procedimentos relatados por Kimura e Miller (1957).

Os parâmetros da cinética de fluxo da fase sólida foram estimados pelo processo iterativo do algoritmo Marquardt, com auxílio do procedimento para modelos não-lineares (PROC NLIN) do programa SAS (User's..., 1985), segundo os modelos descritos por Grovum e Williams (1973) e Dhanoa et al. (1985).

No modelo bicompartimental bi-exponencial de Grovum e Williams (1973), de expressão geral: $\mathrm{Y}=\mathrm{A} * \mathrm{e}^{-\mathrm{k} 1 *(\mathrm{t}-\mathrm{TT})}-\mathrm{A} * \mathrm{e}^{-\mathrm{k} 2 *(\mathrm{t}-\mathrm{TT})}$, para $\mathrm{t} \geq \mathrm{TT}$ e $\mathrm{Y}=0$, para $\mathrm{t}<\mathrm{TT}$, o parâmetro de escala "A" é indefinido do ponto de vista biológico. Os parâmetros " $\mathrm{k}_{1}$ " e " $\mathrm{k}_{2}$ " correspondem, respectivamente, às taxas de passagem no rúmen-retículo e no ceco e cólon proximal, enquanto TT refere-se ao tempo de trânsito no omaso e intestinos delgado e grosso ou, ainda, ao tempo transcorrido desde a dosificação até o primeiro aparecimento do indicador nas fezes. A concentração fecal do indicador no tempo " $t$ " é definida pela variável dependente $\mathrm{Y}$.

Conforme apresentaram Oliveira et al. (1999), a expressão matemática simplificada do modelo multicompartimental de Dhanoa et al. (1985) é:

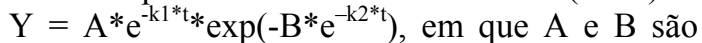
parâmetros de escala, sem definição biológica; e $\mathrm{k}_{1}$ e $\mathrm{k}_{2}$, respectivamente, as taxas de passagem ruminal e pós-ruminal.

Pela inexistência de critério padronizado imposta por sua natureza não-linear, as comparações entre os modelos, depois de verificado o número de curvas passíveis de ajuste e a coerência biológica dos parâmetros estimados (Dhanoa et al., 1985), foram baseadas nos resultados obtidos para os quadrados médios do erro (QME) e nos coeficientes de determinação $\left(\mathrm{R}^{2}\right)$, além do julgamento visual subjetivo da dispersão, no tempo, dos resíduos, em relação aos valores observados (Dhanoa et al., 1985; Beauchemin e Buchanan-Smith, 1989; Oliveira et al., 1999; Detmann et al., 2001).

Foram geradas quatro curvas referentes a cada combinação tratamento $\mathrm{x}$ idade de corte do capim-elefante, considerando, simultaneamente, os dados de excreção fecal das quatro ou três vacas utilizadas, respectivamente, no primeiro e no segundo ano do experimento. Adicionalmente, como subsídio à modelagem comparativa, obtiveram-se 14 curvas de ajuste para cada modelo, aos dados individuais de excreção fecal do indicador na interação tratamento $\mathrm{x}$ vaca, sendo oito no primeiro e seis no segundo ano do experimento.

Ressalte-se que a análise de variância de parâmetros obtidos de equações individuais (rodadas por animal) não tem respaldo estatístico para sua execução (uma vez que todos já são estimativas). Assim, a discussão dos resultados obtidos a partir do ajuste dos dois modelos nãolineares será feita pela comparação direta das estimativas dos parâmetros biológicos que 
representam o fenômeno de fluxo da fase sólida do capim-elefante picado nas condições estudadas, ou seja, suplementado ou não com concentrados em cada idade de corte.

Os cálculos dos tempos de retenção ruminal e pós-ruminal, do tempo médio de retenção no TGI (modelos Grovum e Williams, 1973 e Dhanoa et al., 1985) e do tempo de trânsito (modelo de Dhanoa et al., 1985) foram realizados, segundo procedimentos relatados por Lira et al. (2006).

No primeiro ano do experimento, ao se utilizar o modelo de Grovum e Williams (1973), estimouse também a taxa de passagem da fase sólida do concentrado utilizado na suplementação do capim-elefante picado. Foram colocados no rúmen das vacas que receberam o tratamento com suplementação $80 \mathrm{~g}$ de concentrado mordentado; as coletas de fezes foram realizadas às $6,14,18,25,31,37,40,48,56,64,72,84,90$, 108 e $120 \mathrm{~h}$ pós-dosificação do indicador. Entre os períodos de determinação das taxas de passagem do capim-elefante picado e do concentrado, foi observado intervalo nunca inferior a cinco dias, contados depois do término do período de coleta de fezes, visando assegurar completa exaustão do cromo residual no TGI das vacas.

\section{RESULTADOS E DISCUSSÃO}

No primeiro ano de experimento, as taxas de passagem ruminal $\left(\mathrm{k}_{1}\right)$ e pós-ruminal $\left(\mathrm{k}_{2}\right)$ da fase sólida do concentrado foram de 5,1 e $8,7 \% / \mathrm{h}$, respectivamente. Ao trabalharem com vacas leiteiras que receberam dieta baseada em silagens de milho e de alfafa ( $83 \%$ da MS) e suplemento concentrado composto basicamente por milho laminado e farelo de soja, Colucci et al. (1982) relataram taxas de passagem ruminal e pósruminal, respectivamente, de 6,83 e $15,44 \% / \mathrm{h}$. Aroeira et al. (1995) observaram taxa de passagem ruminal de $7,2 \% / \mathrm{h}$ para o farelo de algodão fornecido para vacas Holandês x Zebu em lactação, e que recebiam cana-de-açúcar adicionada de 1\% da mistura 9:1 uréia:sulfato de amônio (base matéria natural), sendo a relação volumoso:concentrado dessa dieta de 77,5:22,5\% (base MS).

Os parâmetros biológicos da cinética de fluxo de partículas do capim-elefante picado, estimados em função do tratamento experimental (Tab. 1), a partir dos modelos de Grovum e Williams (1973) e de Dhanoa et al. (1985) apresentaram-se dentro da faixa de valores relatados por Lopes et al. (2003), a saber, de 2,1 a $4,1 \% / h$ para $\mathrm{k}_{1}$, e de 3,9 a $11,3 \% / \mathrm{h}$ para $\mathrm{k}_{2}$. Esses autores trabalharam com os modelos de Grovum e Williams (1973) e de Dhanoa et al. (1985) ajustados para dados de excreção fecal de vacas Holandês x Zebu em lactação, manejadas em pastagem de capimelefante com 30 a 45 dias de intervalo de descanso e três dias de ocupação dos piquetes, na estação das chuvas.

Tabela 1. Parâmetros biológicos da cinética de fluxo de partículas do capim-elefante picado, fornecido a vacas Holandês x Zebu em lactação, suplementado ou não com concentrado $(3,3 \mathrm{~kg} / \mathrm{vaca} / \mathrm{dia}$, base matéria natural $)^{\mathrm{a}}$

\begin{tabular}{|c|c|c|c|c|c|c|}
\hline \multirow{3}{*}{ Tratamento } & \multicolumn{6}{|c|}{ Parâmetros da dinâmica da fase sólida do capim-elefante picado ${ }^{b}$} \\
\hline & \multicolumn{3}{|c|}{ Grovum e Williams (1973) } & \multicolumn{3}{|c|}{ Dhanoa et al. (1985) } \\
\hline & $\mathrm{k}_{1}(\% / \mathrm{h})$ & $\mathrm{k}_{2}(\% / \mathrm{h})$ & $\mathrm{R}^{2}$ do modelo & $\mathrm{k}_{1}(\% / \mathrm{h})$ & $\mathrm{k}_{2}(\% / \mathrm{h})$ & $\mathrm{R}^{2}$ do modelo \\
\hline \multicolumn{7}{|c|}{ Capim-elefante com idade de corte de 60 dias } \\
\hline Com concentrado $^{c}$ & 3,18 & 7,37 & 88,1 & 2,97 & 10,94 & 89,2 \\
\hline Sem concentrado ${ }^{\mathrm{d}}$ & 3,06 & 5,81 & 80,3 & 2,82 & 8,75 & 81,7 \\
\hline \multicolumn{7}{|c|}{ Capim-elefante com idade de corte de 45 dias } \\
\hline Com concentrado ${ }^{\mathrm{d}}$ & 3,04 & 4,99 & 89,5 & 2,61 & 8,17 & 91,4 \\
\hline Sem concentrado ${ }^{\mathrm{d}}$ & 3,05 & 4,94 & 90,7 & 2,79 & 7,40 & 92,7 \\
\hline
\end{tabular}

${ }^{a}$ Os dados de excreção fecal do cromo foram ajustados por tratamento, segundo os modelos não-lineares de Grovum e Williams (1973) e de Dhanoa et al. (1985).

${ }^{b} k_{1}=$ taxa de passagem no rúmen; $\mathrm{k}_{2}=$ taxa de passagem no ceco e cólon; $\mathrm{R}^{2}=$ coeficiente de determinação do modelo.

${ }^{\text {c} P a r a ̂ m e t r o s ~ d e ~ f l u x o ~ d e ~ p a s s a g e m ~ d a ~ f a s e ~ s o ́ l i d a, ~ o b t i d o s ~ a ~ p a r t i r ~ d e ~ d a d o s ~ d e ~ e x c r e c ̧ a ̃ o ~ f e c a l ~ d e ~ q u a t r o ~ v a c a s . ~}$

dParâmetros de fluxo de passagem da fase sólida, obtidos a partir de dados de excreção fecal de três vacas. 
Conforme sugerido por Dhanoa et al. (1985), verificou-se que 13 das 14 curvas individuais de excreção fecal do indicador (Tab. 2), ajustadas pelos modelos de Grovum e Williams (1973) e de Dhanoa et al. (1985) apresentaram coerência biológica nas estimativas dos parâmetros de fluxo da fase sólida.

Segundo Ellis et al. (1994), a obtenção de estimativas das taxas de passagem ruminal $\left(\mathrm{k}_{1}\right)$ e pós-ruminal $\left(\mathrm{k}_{2}\right)$ semelhantes $\left(\mathrm{k}_{1} \cong \mathrm{k}_{2}\right)$ é inconsistente com as premissas relacionadas a modelos de dois compartimentos seqüenciais. Esses autores discutiram eventuais condições em que tal situação se estabelece e recomendaram, baseando-se na literatura, que a razão entre esses dois parâmetros $\left(\mathrm{k}_{2} \div \mathrm{k}_{1}\right)$ deveria exceder o valor de 1,5 para que se alcançasse estimativa confiável da taxa de passagem ruminal. Detmann et al. (2001) fizeram uso dessa recomendação em seu estudo. Valores maiores que 1,5 foram verificados na razão entre esses parâmetros para os dois modelos ajustados por tratamento (Tab. 1), sinalizando coerência biológica dos dados obtidos. No entanto, na Tab. 2, foram observados valores menores que 1,5 ao se considerar os dados individuais de taxas de passagem ajustados pelo modelo de Grovum e Williams (1973) para as vacas do tratamento com suplementação concentrada, indicando inconsistência com a hipótese de existência de dois compartimentos seqüenciais.

A despeito de ter havido convergência dos dois modelos para estimativa dos parâmetros de passagem de partículas do capim-elefante com idade de corte de 60 dias, fornecido como alimento exclusivo à vaca 2 (Tab. 2), os valores obtidos foram considerados atípicos em termos biológicos, subjeção respaldada, principalmente, pela desuniformidade na dispersão dos resíduos ordinários e pelo menor valor de $\mathrm{R}^{2}$. Conforme também observaram Lopes et al. (2003), houve aparente retenção do indicador no TGI desta vaca, constatada por concentração fecal do cromo relativamente elevada, mesmo depois de decorridas $120 \mathrm{~h}$ da administração do indicador FDN-cromo-mordente.

Tabela 2. Parâmetros biológicos da cinética de fluxo de partículas do capim-elefante picado, fornecido a vacas Holandês x Zebu em lactação, suplementado $(3,3 \mathrm{~kg} / \mathrm{vaca} /$ dia, base matéria natural) ou não com concentrado $^{\mathrm{a}}$

\begin{tabular}{|c|c|c|c|c|c|c|c|}
\hline \multirow{3}{*}{ Tratamento } & \multirow{3}{*}{ Vaca } & \multicolumn{6}{|c|}{ Parâmetros da dinâmica da fase sólida do capim-elefante ${ }^{b}$} \\
\hline & & \multicolumn{3}{|c|}{ Grovum e Williams (1973) } & \multicolumn{3}{|c|}{ Dhanoa et al. (1985) } \\
\hline & & $\mathrm{k}_{1}(\% / \mathrm{h})$ & $\mathrm{k}_{2}(\% / \mathrm{h})$ & $\mathrm{R}^{2}$ do modelo & $\mathrm{k}_{1}(\% / \mathrm{h})$ & $\mathrm{k}_{2}(\% / \mathrm{h})$ & $\mathrm{R}^{2}$ do modelo \\
\hline \multicolumn{8}{|c|}{ Capim-elefante com idade de corte de 60 dias } \\
\hline Com concentrado & 1 & 3,94 & 8,26 & 97,7 & 3,36 & 12,34 & 97,6 \\
\hline Com concentrado & 2 & 2,94 & 5,36 & 97,2 & 2,48 & 8,82 & 97,9 \\
\hline Com concentrado & 3 & 4,15 & 8,20 & 94,5 & 3,48 & 14,45 & 95,7 \\
\hline Com concentrado & 4 & 3,22 & 5,62 & 95,2 & 3,06 & 7,64 & 97,6 \\
\hline Sem concentrado & 1 & 4,16 & 6,72 & 97,0 & 3,91 & 9,01 & 98,1 \\
\hline Sem concentrado & $2^{c}$ & 1,90 & 3,65 & 80,5 & 1,55 & 5,87 & 84,6 \\
\hline Sem concentrado & 3 & 2,26 & 9,09 & 92,2 & 2,14 & 12,81 & 93,2 \\
\hline Sem concentrado & 4 & 2,47 & 6,07 & 95,8 & 2,97 & 6,53 & 98,3 \\
\hline \multicolumn{8}{|c|}{ Capim-elefante com idade de corte de 45 dias } \\
\hline Com concentrado & 1 & 3,82 & 4,69 & 96,0 & 2,64 & 10,05 & 97,9 \\
\hline Com concentrado & 2 & 3,25 & 4,19 & 95,4 & 2,18 & 9,10 & 96,8 \\
\hline Com concentrado & 3 & 3,55 & 4,44 & 97,4 & 2,95 & 7,87 & 99,1 \\
\hline Sem concentrado & 1 & 3,09 & 5,84 & 97,3 & 3,28 & 11,94 & 92,4 \\
\hline Sem concentrado & 2 & 2,30 & 5,31 & 94,5 & 1,89 & 9,01 & 94,7 \\
\hline Sem concentrado & 3 & 3,40 & 5,38 & 95,6 & 3,13 & 7,98 & 97,7 \\
\hline \multicolumn{8}{|c|}{$\begin{array}{l}\text { as dados de excreção fecal do cromo foram ajustados por vaca, segundo os modelos não-lineares de Grovum } \\
\text { Williams (1973) e de Dhanoa et al. (1985). } \\
\text { b } \mathrm{k}_{1}=\text { taxa de passagem no rúmen; } \mathrm{k}_{2}=\text { taxa de passagem no ceco e cólon; } \mathrm{R}^{2}=\text { coeficiente de determinação do } \\
\text { modelo. }\end{array}$} \\
\hline
\end{tabular}


Os ajustes dos modelos de Grovum e Williams (1973) e de Dhanoa et al. (1985) aos dados de excreção fecal do indicador resultaram em valores de $\mathrm{R}^{2}$ acima de $90 \%$ em 13 das 14 curvas ajustadas por vaca (Tab. 2). No entanto, as curvas obtidas por tratamento (Tab. 1), a despeito de apresentarem menores valores de $\mathrm{R}^{2}$, pois incluíram o efeito animal, representam, de modo mais fidedigno, os parâmetros de fluxo da fase sólida de cada condição estudada (idade de corte do capim e utilização ou não de suplemento concentrado).

Aparentemente, o processo de dependência de tempo imposto pelo tratamento matemático na expressão algébrica do modelo de Dhanoa et al. (1985) permitiu melhor ajuste aos dados de excreção fecal do indicador, em relação ao modelo bicompartimental bi-exponencial tempoindependente de Grovum e Williams (1973). Comportamento semelhante foi relatado por Oliveira et al. (1999) e Lira et al. (2006). Considerando as 10 curvas rodadas por vaca que apresentaram coerência biológica nos parâmetros estimados, além daquelas três cujo valor de $\mathrm{k}_{2} \div$ $\mathrm{k}_{1}$ foi menor que a 1,5 , conforme discutido anteriormente (Tab. 2), 11 foram mias bem ajustadas $\left(>\mathrm{R}^{2}\right.$ e $<$ QME) pelo modelo de Dhanoa et al. (1985). Isso também foi observado nas quatro curvas rodadas por tratamento (Tab. 1). Adicionalmente, de modo subjetivo, pôde-se verificar nessas curvas menor dispersão dos resíduos no ajuste do modelo de Dhanoa et al. (1985) aos dados de excreção fecal.

De fato, esperava-se que o processo de discriminância de tempo implícito no modelo de Dhanoa et al. (1985) garantisse a ele superioridade no ajuste aos dados de excreção fecal do indicador em relação ao modelo de Grovum e Williams (1973), já que a probabilidade de passagem através do orifício retículo-omasal aumenta à medida que a partícula diminui de tamanho, o que está diretamente relacionado ao tempo de estada no rúmen (Offer e Dixon, 2000), em conseqüência de eventos como ruminação, hidratação e degradação microbiana (Quiroz et al., 1988).

De modo geral, as taxas de passagem ruminal e pós-ruminal obtidas do ajuste do modelo de Grovum e Williams (1973) aos dados de excreção fecal do cromo foram, respectivamente, maiores e menores que as estimadas pelo modelo de Dhanoa et al. (1985) (Tab. 1 e 2). Resultados semelhantes foram apresentados por Oliveira et al. (1999) e por Lopes et al. (2003).

Os tempos médios de retenção (TMR) no TGI, calculados a partir dos parâmetros da dinâmica de fluxo da fase sólida do modelo de Dhanoa et al. (1985) foram, de modo geral, menores se comparados aos obtidos do modelo de Grovum e Williams (1973). Para o capim-elefante com 60 dias de crescimento suplementado ou não com concentrados, os valores de TMR calculados foram, respectivamente, de 66,0 e $70,6 \mathrm{~h}$ (Grovum e Williams, 1973) e de 48,1 e 53,7h (Dhanoa et al., 1985). Para o capim-elefante com 45 dias de idade de corte, considerando o modelo de Grovum e Williams (1973), foram observados valores de 76,2 e 75,8h, respectivamente para o tratamento com ou sem suplementação concentrada. Os respectivos valores obtidos a partir do modelo de Dhanoa et al. (1985) foram de 57,8 e $57,7 \mathrm{~h}$. Trabalhando com o modelo de Grovum e Williams (1973), ajustado para dados de excreção fecal de vacas Holandês x Zebu em lactação, manejadas em pastagem de capimelefante com 45 dias de intervalo de descanso e três dias de ocupação dos piquetes, na estação das chuvas, Lopes et al. (2003) relataram valores de TMR variando entre 49,0 e $75,7 \mathrm{~h}$.

\section{CONCLUSÕES}

Foram verificadas diferenças nas taxas de passagem da fase sólida quando estimadas utilizando o modelo multicompartimental tempodependente em comparação ao modelo bicompartimental tempo-independente.

O processo de dependência de tempo imposto pelo modelo multicompartimental permitiu melhor ajuste aos dados de excreção fecal de cromo em vacas Holandês x Zebu alimentadas com capim-elefante picado, em relação ao modelo bicompartimental.

\section{REFERÊNCIAS BIBLIOGRÁFICAS}

AROEIRA, L.J.M.; LOPES, F.C.F.; DAYRELL, M.S. et al. Digestibilidade, degradabilidade e taxa de passagem da cana-de-açúcar mais uréia e do farelo de algodão em vacas mestiças Holandês x Zebu em lactação. Rev. Soc. Bras. Zootec., v.24, p.1016-1026, 1995. 
BEAUCHEMIN, K.A.; BUCHANAN-SMITH, J.G. Evaluation of markers, sampling sites and models for estimating rates of passage of silage or hay in dairy cows. Anim. Feed Sci. Technol., v.27, p.59-75, 1989.

DETMANN, E.; CECON, P.R.; PAULINO, M.F. et al. Estimação de parâmetros da cinética de trânsito de partículas em bovinos sob pastejo por diferentes seqüências amostrais. Rev. Bras. Zootec., v.30, p.222230, 2001.

COLUCCI, P.E.; CHASE, L.E.; VAN SOEST, P.J. Feed intake, apparent diet digestibility, and rate of particulate passage in dairy cattle. J. Dairy Sci., v.65, p.1445-1456, 1982.

DHANOA, M.S.; SIDDONS, R.C.; FRANCE, J. et al. A multicompartimental model to describe marker excretion patterns in ruminant faeces. Br. J. Nutr., v.53, p.663-671, 1985.

EHLE, F.R. Influence of feed particle density on particulate passage from rumen of holstein cow. $J$. Dairy Sci., v.67, p.693-697, 1984.

ELLIS, W.C.; MATIS, J.H.; HILL, T.M. et al. Methodology for estimating digestion and passage kinectics of forage. In: NATIONAL CONFERENCE ON FORAGE QUALITY, EVALUATION, AND UTILIZATION, 1994, Madison. Proceedings... Madison: American Society of Agronomy/Crop Science Society of America/Soil Science Society of America, 1994. p.682-756.

FOX, D.G.; TEDESCHI, L.O.; TYLUTKI, T.P. et al. The net carbohydrate and protein system model for evaluating herd nutrition and nutrient excretion. Anim. Feed Sci. Technol., v.112, p.29-78, 2004.

GROVUM, W.L.; WILLIAMS, V.J. Rate of passage of digesta in sheep. 4. Passage of marker through the alimentary tract and the biological relevance of rateconstants derived from the changes in concentration of marker in faeces. Br. J. Nutr., v.30, p.313-329, 1973.

KIMURA, F.T.; MILLER, V.L. Chromic oxide measurement. Improved determination of chromic oxide in cow feed and feces. J. Agric. Food Chem., v.5, p.216, 1957.

LASCANO, C.; QUIROZ, R. Metodologia para estimar la dinámica de la digestión en rumiantes. In: RUIZ, M.E.; RUIZ, A. (Eds.). Nutrición de rumiantes: Guía metodológica de investigación. San Jose: ALPA/IICA/RISPAL, 1990. p.89-104.

LIRA, V.M.C.; PEREIRA, J.C.; VIEIRA, R.A.M. et al. Avaliação de marcadores e modelos matemáticos para o estudo das cinéticas de trânsito e degradação ruminal em novilhos mantidos em pastagem de capimbraquiária (Brachiaria decumbens Stapf.). Rev. Bras. Zootec., v.35, p.902-913, 2006.

LOPES, F.C.F. Taxa de passagem, digestibilidade in situ, consumo, composição química e disponibilidade de capim-elefante (Pennisetum purpureum, Schumack) pastejado por vacas mestiças Holandês $x$ Zebu em lactação. 2002. 223f. Tese (Doutorado em Ciência Animal) - Escola de Veterinária, Universidade Federal de Minas Gerais, Belo Horizonte.

LOPES, F.C.F.; AROEIRA, L.J.M.; DAYRELL, M.S. et al. Digestibilidade e degradabilidade do capimelefante (Pennisetum purpureum, Schum.) picado, em vacas Holandês x Zebu. In: REUNIÃO ANUAL DA SOCIEDADE BRASILEIRA DE ZOOTECNIA, 34., Juiz de Fora, 1997. Anais... Juiz de Fora: SBZ, 1997. v.1, p.249-252.

LOPES, F.C.F.; RODRIGUEZ, N.M.; AROEIRA, L.J.M. et al. Modelagem comparativa da cinética de fluxo da fase sólida do capim-elefante (Pennisetum purpureum, Schumack) consumido sob pastejo por vacas mestiças Holandês x Zebu em lactação. Arq. Bras. Med. Vet. Zootec., v.55, p.702-709, 2003.

NUTRIENT Requirements of Dairy Cattle. 7. ed. Washington: National Research Council, 2001. 381p.

OFFER, N.W.; DIXON, J. Factors affecting outflow rate from the reticulo-rumen. Nutr. Abs. Rev. (Series B), v.70, p.833-844, 2000.

OLIVEIRA, R.L.; PEREIRA, J.C.; CARVALHO, P.R. et al. Degradabilidade ruminal da cama de frango e do feno de capim Coast-cross e avaliação de modelos matemáticos para estimativa da taxa de passagem de partículas. Rev. Bras. Zootec., v.28, p.839-849 1999 .

QUIROZ, R. A.; POND, K. R.; TOLLEY, E. A. et al. Selection among nonlinear models for rate of passage studies in ruminants. J. Anim. Sci., v.66, p.2977-2986, 1988.

ÚDEN, P.; ROUNSAVILLE, T.R.; WIGGANS, G.R. et al. The mensurement of liquid and solid digesta retention in ruminants, equines and rabbits given timothy (Phleum pratense) hay. Br. J. Nutr., v.48, p.329-339, 1982.

USER'S guide: statistics. Cary, NC: SAS Institute, 1985. 956p.

VAN SOEST, P.J. Nutritional ecology of the ruminant. 2.ed. Ithaca: Cornell University Press, 1994. 476p. 